\title{
Role of aerobic glycolysis in genetically engineered mouse models of cancer
}

\author{
Chi V Dang* \\ See research article: http://www.cancerandmetabolism.com/content/1/1/2
}

\begin{abstract}
The propensity of cancer cells to convert high levels of glucose to lactate through aerobic glycolysis has been intensively studied in vitro, and is now understood to be a metabolic adaptation that shunts glucose carbons toward building blocks for the growing cell, as well as producing ATP. Much less is known, however, about the role of aerobic glycolysis and glycolytic enzymes in vivo. A paper in Cancer and Metabolism now documents aerobic glycolysis in the proliferating neural progenitors that form the cerebellum in normal newborn mice, as well as in medulloblastoma tumors derived from these cells in transgenic mice. Hexokinase $\|$ is demonstrated to be an essential driver of the observed aerobic glycolysis and the malignancy of the tumors.
\end{abstract}

\section{Why cancer has renewed interest in glycolysis}

Glucose is a key bioenergetic substrate for mammals, and the steps by which it is metabolized in cells to produce energy in the form of ATP were worked out long ago. As shown in Figure 1, these start with a series of chemical conversions to pyruvate (producing two ATPs) followed either by oxidation in the mitochondrion (producing 30 ATPs) or the conversion of pyruvate to lactate. The steps from glucose to lactate constitute glycolysis, and from the original studies in muscle and other tissues, it was concluded that the main function of this pathway was to supply pyruvate for mitochondrial respiration to meet the energy demands of the cell. Conversion to lactate was observed only in hypoxic conditions and anaerobic glycolysis viewed as a temporary and much less efficient way to produce ATP.

\footnotetext{
*Correspondence: dangvchi@upenn.edu

Abramson Cancer Center, University of Pennsylvania, Perelman School of Medicine, Philadelphia, Pennsylvania 19104, USA
}

This view changed when Otto Warburg noted that cancer cells convert glucose to lactate at a high rate in normoxic conditions, a phenomenon that became known as the Warburg effect. Having established that this elevated level of aerobic glycolysis was not due to mitochondrial defects, but an altered metabolism that had advantages for cancer cells, there was a renewed interest in studying the detailed regulation of glycolysis and its connected pathways. This continues today, with in vitro studies still adding to our understanding of the role of glycolysis in meeting the biosynthetic as well as energetic needs of cancer cells [1]. Addressing the role of glycolysis as tumors arise in vivo is challenging, but genetic engineering in mouse models can make a contribution. In this regard, the work of Gershon et al. [2] on hexokinase-2 (HK2)-mediated aerobic glycolysis during cerebellar neurogenesis and the pathogenesis of medulloblastoma is significant, both as an in vivo confirmation of an important role for HK2-driven aerobic glycolysis in tumorigenesis, and in demonstrating a role for aerobic glycolysis in normal development.

\section{Aerobic glycolysis provides anabolic carbons for biosynthetic pathways}

Glucose uptake into normal cells is tightly regulated, but cancer cells are reprogrammed to fuel unrestrained proliferation [1] and diverse cancer types have been observed to take up glucose at abnormally high rates. Once transported across the cell membrane, glucose is trapped by hexokinase-mediated phosphorylation to form glucose-6-phosphate. In non-dividing cells this is mediated by HK1, but HK2 is induced in proliferating cells and is directly activated at the transcriptional level by c-Myc and hypoxia inducible factor (HIF-1) [1]. HK2 binds to the outer mitochondrial membrane and catalyzes the phosphorylation of glucose, putatively by using ATP generated by the mitochondrion. Unlike HK1 it is not inhibited by its product and can therefore drive a higher level of glycolysis. The phosphorylation of glucose is a key step that defines the uptake and retention of 


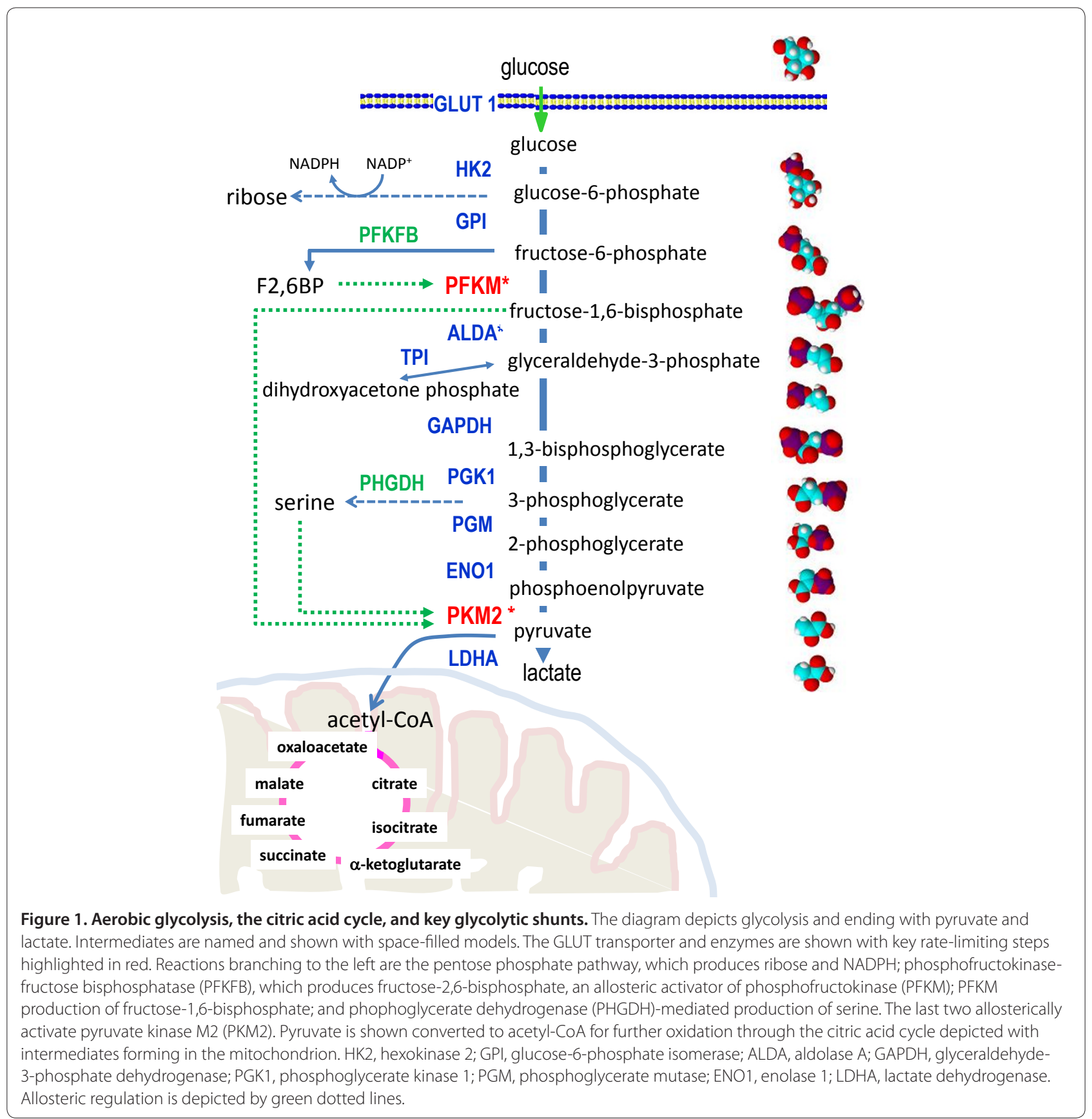

positron emitting ${ }^{19} \mathrm{~F}$-fluorodeoxyglucose in clinical PET scans of cancers. As well as being useful diagnostically, PET scans provide an in vivo demonstration of high glucose consumption by human tumors in situ.

Several glycolytic steps between HK2 and the final enzyme in the pathway, LDHA, are noteworthy as important regulatory points (Figure 1). Phosphofructokinase (PFK) is a rate-limiting, allosterically controlled enzyme that catalyzes the conversion of fructose-6phosphate to fructose-1,6-bisphosphate (F1,6BP) before its conversion to 3-carbon molecules and ultimately to pyruvate. Regulation of PFK by the allosteric activator fructose-2,6-bisphosphate (F2,6BP), which is generated by the 6-phosphofructo-2-kinase/fructose-2,6-biphosphatase family, provides a means to increase glycolytic flux and this has been shown to be important for prostate cancer cell survival [3]. Alternatively, a choke point is created, when PFK activity diminishes, to shunt glucose carbons toward the pentose phosphate pathway for NADPH generation and redox homeostasis, which are critical for titrating oxygen radicals generated by mitochondrial respiration 
The other notable step involves pyruvate kinase $M$ (PKM), which catalyzes the conversion of phosphoenolpyruvate to pyruvate and the generation of ATP. The rate-limiting PKM2 isoform, resulting from a specific spliced mRNA form, is under allosteric control of F1,6BP and serine that is generated from 3PG (Figure 1) [4]. These mechanisms of control of PFK and PKM2 provide key shunts for channeling glucose carbons toward biosynthetic pathways or for redox homeostasis. Finally, the conversion of pyruvate to lactate is coupled with the recycling of $\mathrm{NADH}$ to $\mathrm{NAD}^{+}$, which is required for glyceraldehyde phosphate dehydrogenase (GAPDH) upstream in the glycolytic pathway. It appears that this recycling of $\mathrm{NAD}^{+}$ with the dumping of glucose carbons as lactate is essential for cellular redox homeostasis, because inhibition of LDHA results in oxidative stress-induced cell death. Similar to mammalian cells, proliferating yeast cells dump glucose as ethanol as a way to recycle $\mathrm{NAD}^{+}$.

\section{Essential role of hexokinase in tumorigenesis}

Although studies have demonstrated an essential role of aerobic glycolysis for cultured cell proliferation and LDHA for tumor xenograft growth, the role of HK2 in mouse tumorigenesis had not been previously established. Gershon et al. [2] document that HK2 is required for medulloblastoma tumorigenesis driven by a constitutively active sonic hedgehog (Shh) pathway in cerebellar granule neuron progenitor (CGNP) cells. Stimulation of isolated CGNP cells with Shh resulted in increased aerobic glycolysis and activation of $\mathrm{HK} 2$ in an N-Myc-Max-dependent manner, suggesting that Shh pathway-mediated increase in Myc activity drives HK2 expression and glycolysis, which are both accentuated by HIF-1 under hypoxia. Importantly, conditional deletion of HK2 diminishes Shh-mediated aerobic glycolysis in CGNPs, indicating that normal development in this lineage involves glycolysis, which is commandeered for tumorigenesis. In this regard, Gershon et al. [2] documented that deletion of $\mathrm{HK} 2$ disrupted medulloblastoma tumorigenesis and prolonged survival.

\section{Complex role of lactate dehydrogenases in cancer}

By contrast to HK2, the role of LDHs in cancer development appears more complex. Although targeting LDHA with short-hairpin RNAs (shRNAs) diminishes tumor xenograft growth in a number of models, the reduction of LDHA activity in a hypomorphic mutant allele of LDHA did not diminish tumorigenesis in the $\lambda$-Myc transgenic model of murine lymphoma [5]. Although LDHA kinetically favors the conversion of pyruvate to lactate in aerobic glycolysis and is therefore important for tumorigenesis, LDHB could also mediate the same reaction, although it is more sensitive to substrate (pyruvate) inhibition and hence is thought to favor the conversion of lactate to pyruvate. Both LDHs can also catalyze the conversion of lactate to pyruvate, which could be oxidized in the mitochondrion and may be important for the commensal metabolic relationship among cancer cells in the tumor microenvironment. In this relationship, glycolytic hypoxic cancer cells convert glucose to lactate, which is taken up by cells that convert lactate to pyruvate for oxidation by mitochondrial respiration in oxygenated areas of the tumor [6]. Normal stromal cells in the tumor might also participate in this relationship. Indeed, oxidative phosphorylation appears to be critically important for certain cancers and in particular for a subset of human lymphomas [7]. LDHB rather than LDHA appears to be necessary for triple negative breast cancer cell growth [8]. Hence, the roles of LDHA and LDHB in aerobic glycolysis or in mitochondrial respiration in specific types of human cancers remain to be fully established.

\section{Don't forget about the mitochondrion and other nutrient sources}

As already alluded to, mitochondrial respiration is essential for certain tumors to grow and progress. Further, the mitochondrion is a hotbed for many essential biochemical pathways used by growing cells, such as pyrimidine, amino acid and heme biosynthesis [9]. Thus, although aerobic glycolysis plays an important role in tumorigenesis, the role of the mitochondrion should not be forgotten. For example, the commensal roles of hypoxic and aerobic cancer cells require functional mitochondria to use lactate as a respiratory substrate [6]. In addition, fatty acids, rather than glucose, could be oxidized as a primary bioenergetics source for lymphoma and leukemia cells [7]. Aerobic glycolysis and mitochondria provide ATP and building blocks for cancer cells, when nutrients are available. Cancer cells starved of nutrients could survive and proliferate by eating cellular components via autophagy or adapt by eating macromolecules from their environment by macropinocytosis [10].

In summary, the work of Gershon et al. [2] definitively documents a role for HK2 and aerobic glycolysis for the generation of medulloblastoma in mice and underscores the importance of the Warburg effect in tumorigenesis . However, the loss of HK2 prolonged survival but did not fully block tumorigenesis, suggesting that tumorigenesis could rely on alternative metabolic pathways. Studies of tumors arising from targeted deletion of HK2 could lead to additional insights into metabolic reprogramming in tumorigenesis and the role of the tumor microenvironment.

\footnotetext{
Acknowledgements

This work is supported in part by the National Cancer Institute, Leukemia \& Lymphoma Society, and the Abramson Family Cancer Research Institute.
}

Published: 23 January 2013 


\section{References}

1. Cantor JR, Sabatini DM: Cancer cell metabolism: one hallmark, many faces. Cancer Discov 2012, 2:881-898.

2. Gershon TR, Crowther AJ, Tikunov A, Garcia I, Annis R, Yuan H, Miller CR, MacDonald J, Olson J, Deshmukh M: Hexokinase-2-mediated aerobic glycolysis is integral to cerebellar neurogenesis and pathogenesis of medulloblastoma. Cancer Metab 2013, 1:2.

3. Ros S, Santos CR, Moco S, Baenke F, Kelly G, Howell M, Zamboni N, Schulze A: Functional metabolic screen identifies 6-phosphofructo-2-kinase/ fructose-2,6-biphosphatase 4 as an important regulator of prostate cancer cell survival. Cancer Discov 2012, 2:328-343.

4. Chaneton B, Hillmann P, Zheng L, Martin AC, Maddocks OD, Chokkathukalam A, Coyle JE, Jankevics A, Holding FP, Vousden KH, Frezza C, O'Reilly M, Gottlieb E: Serine is a natural ligand and allosteric activator of pyruvate kinase M2. Nature 2012, 491:458-462

5. Nilsson LM, Forshell TZ, Rimpi S, Kreutzer C, Pretsch W, Bornkamm GW, Nilsson JA: Mouse genetics suggests cell-context dependency for Mycregulated metabolic enzymes during tumorigenesis. PLoS Genet 2012, 8:e1002573.

6. Sonveaux P, Végran F, Schroeder T, Wergin MC, Verrax J, Rabbani ZN, De Saedeleer CJ, Kennedy KM, Diepart C, Jordan BF, Kelley MJ, Gallez B, Wahl ML, Feron O, Dewhirst MW: Targeting lactate-fueled respiration selectively kills hypoxic tumor cells in mice. J Clin Invest 2008, 118:3930-3942.
7. Caro P, Kishan AU, Norberg E, Stanley IA, Chapuy B, Ficarro SB, Polak K, Tondera D, Gounarides J, Yin H, Zhou F, Green MR, Chen L, Monti S, Marlo JA, Shipp MA, Danial NN: Metabolic signatures uncover distinct targets in molecular subsets of diffuse large B cell lymphoma. Cancer Cell 2012, 16:547-560.

8. McCleland ML, Adler AS, Shang Y, Hunsaker T, Truong T, Peterson D, Torres E, Li L, Haley B, Stephan JP, Belvin M, Hatzivassiliou G, Blackwood EM, Corson L, Evangelista M, Zha J, Firestein R: An integrated genomic screen identifies LDHB as an essential gene for triple-negative breast cancer. Cancer Res 2012, 15:5812-5823.

9. Koppenol WH, Bounds PL, Dang CV: Otto Warburg's contributions to current concepts of cancer metabolism. Nat Rev Cancer 2011, 11:325-337.

10. Lim JP, Gleeson PA: Macropinocytosis: an endocytic pathway for internalising large gulps. Immuno/ Cell Biol 2011, 89:836-843.

doi:10.1186/1741-7007-11-3

Cite this article as: Dang CV: Role of aerobic glycolysis in genetically engineered mouse models of cancer. BMC Biology 2013, 11:3. 